PHYSICAL REVIEW D 82, 129901(E) (2010)

\title{
Erratum: Extended Hořava gravity and Einstein-aether theory [Phys. Rev. D 81, 101502 (2010)]
}

Ted Jacobson

(Received 11 November 2010; published 1 December 2010)

DOI: 10.1103/PhysRevD.82.129901

PACS numbers: 04.50.Kd, 04.20.Fy, 99.10.Cd

The first line in Eq. (10) should $\operatorname{read} G_{H} / G_{\mathfrak{x}}=\xi=1 /\left(1-c_{13}\right)$. 